\title{
Mamiellophyceae shift in seasonal predominance in the Baltic Sea
}

\section{Majaneva, Markus}

2019-08-22

Majaneva, M , Enberg , S, Autio , R , Blomster , J \& Rintala , J-M 2019 , ' Mamiellophyceae shift in seasonal predominance in the Baltic Sea ' , Aquatic Microbial Ecology , vol. 83 , no. 2 , pp. 181-187 . https://doi.org/10.3354/ame01915

http://hdl.handle.net/10138/318478

https://doi.org/10.3354/ame01915

CC BY

acceptedVersion

Downloaded from Helda, University of Helsinki institutional repository.

This is an electronic reprint of the original article.

This reprint may differ from the original in pagination and typographic detail.

Please cite the original version. 


\title{
Mamiellophyceae shift in seasonal predominance in the Baltic Sea
}

\author{
M. Majaneva ${ }^{1,2, *}$, S. Enberg ${ }^{2,3}$, R. Autio ${ }^{4}$, J. Blomster ${ }^{3}$, J.-M. Rintala ${ }^{2,3,5}$ \\ ${ }^{1}$ Department of Natural History, NTNU University Museum, Norwegian University of \\ Science and Technology, 7491 Trondheim, Norway \\ ${ }^{2}$ Tvärminne Zoological Station, University of Helsinki, 10900 Hanko, Finland \\ ${ }^{3}$ Faculty of Biological and Environmental Sciences, 00014 University of Helsinki, Finland \\ ${ }^{4}$ Marine Research Centre, Finnish Environment Institute, 00790 Helsinki, Finland \\ ${ }^{5}$ Institute for Atmospheric and Earth System Research (INAR), Faculty of Science, 00014 \\ University of Helsinki, Finland \\ *Corresponding author: markus.majaneva@ntnu.no
}

Running page head: Majaneva et al.: Mamiellophyceae succession

\begin{abstract}
The green algae Mamiellophyceae are a group of marine picoeukaryotes. We studied the succession of Mamiellophyceae in the Baltic Sea water column and ice from autumn to summer, using the hypervariable V4 region of the $18 \mathrm{~S}$ ribosomal RNA gene. We show that species of Mamiellophyceae shift in seasonal predominance and that different species characterize sea ice, under-ice water and the water column in the Baltic Sea.
\end{abstract}

KEY WORDS: 18S rRNA gene · Hypervariable V4 region · Green algae · Picoeukaryotes · Succession

\section{Introduction}

Mamiellophyceae, a class of green algae, includes 3 described orders: Mamiellales, Dolichomastigales and Monomastigales (Marin \& Melkonian 2010). Dolichomastigales and Monomastigales host many lineages, but they are not abundant in marine waters (Monier et al. 2016). In contrast, the most common Mamiellophyceae in coastal waters-species from the Mamiellales genera Ostreococcus, Bathycoccus and Micromonas - may contribute significantly to the primary production of picoeukaryotes (Worden et al. 2004, Tragin \& Vaulot 2018). This has raised research interest in Mamiellophyceae recently, and with the help of molecular methods, the contributions and seasonal patterns of different lineages have become possible to investigate in more detail (e.g. Foulon et al. 2008, Demir-Hilton et al. 2011).

Mamiellophyceae as a class does not show preferences to environmental conditions globally (Tragin \& Vaulot 2018), and Mamiellophyceae biogeography is driven largely by geographical location rather than water depth (Monier et al. 2016). For example, species of Ostreococcus are globally distributed but are not always found together with usually cooccurring Bathycoccus and Micromonas (Demir-Hilton et al. 2011), and Ostreococcus species are absent in Arctic waters (Tragin \& Vaulot 2019). Ostreococcus is, however, present in adjacent seasonally sea ice covered areas, for example the Baltic Sea and the White Sea (Majaneva et al. 2012, Belevich et al. 2018).

At the species and genetic strain level, Mamiellophyceae occupy different ecological niches. For example, within the morphospecies Micromonas pusilla, different genetic lineages recently divided into several species (Simon et al. 2017) show shifts in abundance along local and basin-wide environmental gradients (Foulon et al. 2008). Similarly, Ostreococcus strains show differences in distribution, and co-occurrence at the same geographical location is rare 
(Demir-Hilton et al. 2011). Metagenomes of the genetically less variable Bathycoccus prasinos indicate the same (Vannier et al. 2016).

In the Baltic Sea, species of Monomastix, Dolichomastix, Mamiella, Mantoniella and Micromonas are reported (Hällfors 2004, Majaneva et al. 2012). Mantoniella sp. appears characteristic for ice, while B. prasinos is characteristic for under-ice water (Majaneva et al. 2017). However, there are no studies on seasonal patterns or environmental preferences of Mamiellophyceae in the Baltic Sea. Here, we take advantage of our bi-weekly sampling of cold water season protists and describe Mamiellophyceae succession based on V4 reads of the 18S ribosomal RNA (18S rRNA) gene in Baltic Sea water and sea ice during the period from autumn to summer.

\section{Materials and Methods}

Samples were collected from 2 coastal brackish water (salinity 3.5-6) sites in the northwestern Gulf of Finland, Baltic Sea. Sampling was carried out from 8 October 2012 to 8 May 2013, with extra samples on 3 September 2012 and 1 July 2013. The sampling protocol, DNA extraction, PCR setup and sequencing are described in Enberg et al. (2018) and in Supplement 1 at www.int-res.com/articles/suppl/mXXXpXXX_supp/. Enberg et al. (2018) outlined the eukaryotic communities using microscopy and a 310 bp long fragment of the V4 region of the $18 \mathrm{~S}$ rRNA gene (hereafter short V4). Here, we focus on Mamiellophyceae short $\mathrm{V} 4$ reads and a longer fragment of the V4 region that was amplified using $574 * \mathrm{~F}$ and $1132 \mathrm{R}$ (Hugerth et al. 2014) primers (hereafter long V4). This long V4 was approximately 540 bp long. The raw reads are available at the ENA SRA repository with the study names PRJEB21047, PRJEB23628 and PRJEB25089.

The paired-end sequenced short and long V4 reads were merged using the fastq_mergepairs command in vsearch v.2.6.2 (Rognes et al. 2016). The quality filtering using the -fastq_filter command discarded reads shorter than 320 bases (short V4) and 550 bases (long V4), reads longer than 380 bases (short V4) and 610 bases (long V4), reads with ambiguous bases and reads with over 1 maximum expected error. Primer sequences were removed using the command trim.seqs in mothur v.1.39.5 (Schloss et al. 2009). Exact duplicates were removed using the command -derep_fulllength in vsearch. Chimeric reads were searched using the command -uchime_denovo in vsearch. A read was treated as chimeric and removed if its abundance was 5 times lower than its assumed parental reads. Operational taxonomic units (OTUs) were searched using the option fastidious in swarm v.2.1.6 (Mahé et al. 2015). The abundance of each OTU in each sample was searched using the -usearch_global command (-id 0.0) in vsearch.

The OTUs were assigned taxonomically searching all OTUs first against the NCBI non-redundant nucleotide database (short V4: 9 February 2018, long V4: 10 February 2018) and second against the SILVA_132_SSURef database (Quast et al. 2013), using BLAST v.2.6.0+ (Zhang et al. 2000). All OTUs affiliated with Mamiellophyceae were selected for the subsequent analyses. Read abundance was normalized to the total number of Mamiellophyceae reads in each sample.

To place the OTUs phylogenetically, we took all available sequences of described Mamiellophyceae species together with some relevant uncultured sequences (e.g. Mamiellophyceae DSGM-81) and 6 Pyrenomonadaceae sequences as an outgroup from the SILVA and NCBI Nucleotide databases (23 January 2019). The datasets were combined and aligned with the MAFFT online service (Katoh et al. 2017), and the alignment was cut to the length of the long V4. Bayesian phylogenetic analysis was performed with MrBayes v.3.2.6 (Ronquist et al. 2012). Two independent runs with 4 Markov chains and 1500000 generations 
were carried out. We did not choose the model prior to the analysis but sampled across the general time-reversible model space with gamma-distributed rate variation across sites and a proportion of invariable sites. The resulting estimates (e.g. tree topology) were posterior probability weighted averages of the models. The scripts for methods are provided in Supplement 2. The reads and read abundance are provided in Table S1 in Supplement 3.

\section{Results and Discussion}

In the dataset, 59 short and 46 long V4 Mamiellophyceae OTUs were present, and after manually checking the alignment and combining identical short and long V4 OTUs, 59 OTUs were used in the analyses. Mamiellophyceae OTU richness was significantly lower in sea ice than in under-ice water and the water column (Kruskal-Wallis $\chi^{2}=13.7, p=0.003$, followed by pairwise comparisons using the Mann-Whitney $U$-test). Mamiellophyceae contributed $3.3 \%$ of the total abundance of taxonomically assigned OTUs, and they were present in all samples. The lowest Mamiellophyceae read abundance was in the Krogarviken April ice sample $(<0.1 \%$ of total abundance), and the highest contribution was in the Storfjärden March bottom water sample (17\% of total abundance). Mamiellophyceae read abundance was significantly lower in sea ice and under-ice water than in the water column (Kruskal-Wallis $\chi^{2}=26.3, \mathrm{p}<0.001$, followed by pairwise comparisons using the MannWhitney $U$-test).

The Bayesian phylogenetic tree (Fig. 1 ) resembled the latest phylogenies of Mamiellophyceae (Marin \& Melkonian 2010, Monier et al. 2016, Simon et al. 2017, Yau et al. unpubl.; preprint doi:10.1101/506915), and the OTUs represented all 3 described orders with 35 Mamiellales OTUs, 23 Dolichomastigales OTUs and 1 Monomastigales OTU (Fig. 1). OTUs that referred to the genus Mamiella were not found. In accordance with an earlier global study (Monier et al. 2016), Dolichomastigales were diverse but rare in the Baltic Sea, and the abundant OTUs (>100 reads in total, 22 OTUs) were mainly from Mamiellales. The abundant OTUs were associated with Bathycoccus ( 86923 reads in total, 3 OTUs), Ostreococcus ( 59313 reads, 3 OTUs), Micromonas (36640 reads, 4 OTUs) and Mantoniella (25982 reads, 7 OTUs). The rest of the abundant OTUs were affiliated with DSGM-81 Mamiellophyceae (1147 reads, 3 OTUs) and with Crustomastix (362 reads, 2 OTUs) from Dolichomastigales.

Different Mamiellophyceae OTUs characterized sea ice, under-ice water and the water column (generalized discriminant analysis of 4 first principal coordinates analysis axes, analysis based on $\ln [\mathrm{x}+1]$ transformed Bray-Curtis dissimilarities, squared correlations 0.80 and $0.58, \mathrm{p}<0.001$, misclassification error $43.9 \%$ ).

The Mamiellophyceae OTU associated with an uncultured Baltic Sea ice clone FN690723 characterized sea ice (Fig. 2a,d, Otu07). This species is clearly sea ice associated, and it is present in the sea ice of the Gulf of Bothnia and the Gulf of Finland in the Baltic Sea (Majaneva et al. 2012) and in the White Sea (Belevich et al. 2018). Otu07 and the uncultured Baltic Sea ice clones form a clade of their own, basal to the Mantoniella and Micromonas clade in our Bayesian phylogenetic tree (Fig. 1) but which are classified as Mantoniella in Tragin \& Vaulot (2019). Mantoniella squamata (Otu14) was the most abundant Mantoniella species in the water column in September and October, as was M. beaufortii (Otu04) in November to May and OTUs close to M. antarctica (Otu21, Otu22) in July (Fig. 3c). M. beaufortii and several Crustomastix OTUs characterized under-ice water. The presence of freshwater Crustomastix species in low abundance in under-ice water is an indication of river discharge under ice in the area (Kaartokallio et al. 2007). 
The abundant Bathycoccus, Ostreococcus and Micromonas species characterized the water column but shifted in predominance during the sampling season (Fig. 2c,f,g). Ostreococcus mediterraneus (Otu02) was the most abundant species in October to December, although Bathycoccus and Micromonas species had a relatively high abundance as well. Otu01, closely related to Bathycoccus prasinos, predominated distinctly in January to April, Micromonas polaris (Otu06) in May and M. commoda (Otu03) in July (Fig. 2c,f).

The 4 species of Micromonas alternated in dominance in the water column: the predominant species was M. pusilla (Otu09) in October surface water and M. bravo (Otu10) in bottom water, M. polaris (Otu06) in January to May and M. commoda (Otu03) in summer and early autumn water (Fig. 3a,b). These shifts in predominance are in line with the thermal niches of Micromonas species (Demory et al. 2019).

The 2 abundant Ostreococcus OTUs-O. mediterraneus (Otu02) and the Otu05 basal to the clade of $O$. tauri and $O$. lucimarinus - co-occurred in autumn, and both OTUs were practically absent after March (Fig. 2). The co-occurrence is in accordance with the earlier findings of co-occurring coastal Ostreococcus strains (Demir-Hilton et al. 2011) and the absence of Ostreococcus in cold polar waters (Tragin \& Vaulot 2019). B. prasinos had a similar occurrence in the Baltic Sea, being present and abundant only in the autumn (e.g. Fig. 2c). The 2 Ostreococcus OTUs and B. prasinos appear to be characteristic species of the autumn water community (Enberg et al. 2018).

The most abundant OTU in our dataset (Otu01, 1 base difference to clone FN690721 from Majaneva et al. 2012) was present in all samples. It is closely related to B. prasinos (Fig. 1 ), which is the only described species within the genus currently and whose cultures show identical 18S rRNA gene sequences but differing genomes (Vannier et al. 2016, Tragin \& Vaulot 2019). It is beyond this note, but the uncorrected interspecific 18S rRNA sequence divergence within the other Mamiellales genera is 1.1 to $5.6 \%$, and the $1.1 \%$ uncorrected interspecific divergence of Otu01 and B. prasinos implies that Otu01 could be an uncultured, undescribed species of Bathycoccus.

Here, we have shown that species of Mamiellophyceae shift in seasonal predominance in a coastal brackish water locality in the Baltic Sea and that different species characterize sea ice, under-ice water and the water column during the sea ice covered season. Our findings support the earlier research showing geographic niche partition within Mamiellophyceae (Foulon et al. 2008, Demir-Hilton et al. 2011, Monier et al. 2016) and show that the niche partition holds also for populations in the water and sea ice of the Baltic Sea.

Acknowledgements. Walter and Andrée de Nottbeck Foundation and University of Helsinki 3 yr research grants provided funding to carry out the field and laboratory analysis for this study.

\section{LITERATURE CITED}

<jrn>Belevich TA, Ilyash LV, Milyutina IA, Logacheva MD, Goryunov DV, Troitsky AV (2018) Photosynthetic picoeukaryotes in the land-fast ice of the White Sea, Russia. Microb Ecol 75:582-597 doi:10.1007/s00248-017-1076-x </jrn>

$<$ jrn>Demir-Hilton E, Sudek S, Cuvelier ML, Gentemann CL, Zehr JP, Worden AZ (2011) Global distribution patterns of distinct clades of the photosynthetic picoeukaryote Ostreococcus. ISME J 5:1095-1107 doi:10.1038/ismej.2010.209</jrn>

$<$ jrn>Demory D, Baudoux AC, Monier A, Simon N and others (2019) Picoeukaryotes of the Micromonas genus: sentinels of a warming ocean. ISME J 13:132-146 doi:10.1038/s41396-018-0248-0 </jrn> 
<jrn>Enberg S, Majaneva M, Autio R, Blomster J, Rintala JM (2018) Phases of microalgal succession in sea ice and the water column in the Baltic Sea from autumn to spring. Mar Ecol Prog Ser 599:19-34 doi:10.3354/meps12645</jrn>

$<$ jrn>Foulon E, Not F, Jalabert F, Cariou T, Massana R, Simon N (2008) Ecological niche partitioning in the picoplanktonic green alga Micromonas pusilla: evidence from environmental surveys using phylogenetic probes. Environ Microbiol 10:2433-2443 doi:10.1111/j.1462-2920.2008.01673.x </jrn>

$<$ jrn>Hällfors G (2004) Checklist of Baltic Sea phytoplankton species (including some heterotrophic protistan groups). Baltic Sea Environ Proc 95. Baltic Marine Environment Protection Commission, Helsinki Commission, Helsinki</jrn>

<jrn>Hugerth LW, Muller EEL, Hu YOO, Lebrun LAM and others (2014) Systematic design of 18S rRNA gene primers for determining eukaryotic diversity in microbial consortia. PLOS ONE 9:e95567 doi:10.1371/journal.pone.0095567</jrn>

<jrn>Kaartokallio H, Kuosa H, Thomas DN, Granskog MA, Kivi K (2007) Biomass, composition and activity of organism assemblages along a salinity gradient in sea ice subjected to river discharge in the Baltic Sea. Polar Biol 30:183-197 doi:10.1007/s00300$\underline{006-0172-\mathrm{z}}</ \mathrm{jrn}>$

<jrn>Katoh K, Rozewicki J, Yamada KD (2017) MAFFT online service: multiple sequence alignment, interactive sequence choice and visualization. Brief Bioinform 30:bbx108</jrn> doi:10.1093/bib/bbx108

<jrn>Mahé F, Rognes T, Quince C, de Vargas C, Dunthorn M (2015) Swarm v2: highlyscalable and high-resolution amplicon clustering. PeerJ 3:e1420 doi: $10.7717 /$ peerj. $1420</ j r n>$

<jrn>Majaneva M, Rintala JM, Piisilä M, Fewer PD, Blomster J (2012) Comparison of wintertime nano-sized eukaryotic communities in the Baltic Sea ice and water, based on sequencing of the 18S rRNA gene. Polar Biol 35:875-889 doi:10.1007/s00300-011-1132$\underline{9}</ \mathrm{jrn}>$

<jrn>Majaneva M, Blomster J, Müller S, Autio R and others (2017) Sea-ice eukaryotes of the Gulf of Finland, Baltic Sea, and evidence for herbivory on weakly shade-adapted ice algae. Eur J Protistol 57:1-15 doi:10.1016/j.ejop.2016.10.005</jrn>

$<$ jrn>Marin B, Melkonian M (2010) Molecular phylogeny and classification of the Mamiellophyceae class. nov. (Chlorophyta) based on sequence comparisons of the nuclear- and plastid-encoded rRNA operons. Protist 161:304-336 doi:10.1016/j.protis.2009.10.002</jrn>

$<$ jrn>Monier A, Worden AZ, Richards TA (2016) Phylogenetic diversity and biogeography of the Mamiellophyceae lineage of eukaryotic phytoplankton across the oceans. Environ Microbiol Rep 8:461-469 doi:10.1111/1758-2229.12390</jrn>

<jrn>Quast C, Pruesse E, Yilmaz P, Gerken J and others (2013) The SILVA ribosomal RNA gene database project: improved data processing and web-based tools. Nucleic Acids Res 41:D590-D596 doi:10.1093/nar/gks1219 </jrn>

<jrn>Rognes T, Flouri T, Nichols B, Quince C, Mahé F (2016) VSEARCH: a versatile open source tool for metagenomics. PeerJ 4:e2584 doi:10.7717/peerj.2584</jrn>

<jrn>Ronquist F, Teslenko M, van der Mark P, Ayres DL and others (2012) MrBayes 3.2: efficient Bayesian phylogenetic inference and model choice across a large model space. Syst Biol 61:539-542 doi:10.1093/sysbio/sys029</jrn> 
<jrn>Schloss PD, Westcott SL, Ryabin T, Hall JR and others (2009) Introducing mothur: open-source, platform-independent, community-supported software for describing and comparing microbial communities. Appl Environ Microbiol 75:7537-7541 doi:10.1128/AEM.01541-09</jrn>

$<$ jrn>Simon N, Foulon E, Grulois D, Six C and others (2017) Revision of the genus Micromonas Manton et Parke (Chlorophyta, Mamiellophyceae), of the type species $M$. pusilla (Butcher) Manton \& Parke and of the species M. commoda van Baren, Bachy and Worden and description of two new species based on the genetic and phenotypic characterization of cultured isolates. Protist 168:612-635 doi:10.1016/j.protis.2017.09.002</jrn>

$<$ jrn> Tragin M, Vaulot D (2018) Green microalgae in marine coastal waters: the Ocean Sampling Day (OSD) dataset. Sci Rep 8:14020 doi:10.1038/s41598-018-32338-w</jrn>

$<$ jrn>Tragin M, Vaulot D (2019) Novel diversity within marine Mamiellophyceae (Chlorophyta) unveiled by metabarcoding. Sci Rep 9:5190 doi:10.1038/s41598-019$\underline{41680-6}<$ jirn>

$<$ jrn> Vannier T, Leconte J, Seeleuthner Y, Mondy S and others (2016) Survey of the green picoalga Bathycoccus genomes in the global ocean. Sci Rep 6:37900 doi:10.1038/srep37900</jrn>

$<$ jrn>Worden AZ, Nolan JK, Palenik B (2004) Assessing the dynamics and ecology of marine picophytoplankton: the importance of the eukaryotic component. Limnol Oceanogr 49:168-179 doi:10.4319/1o.2004.49.1.0168</jrn>

$<$ jrn > Zhang Z, Schwartz S, Wagner L, Miller W (2000) A greedy algorithm for aligning DNA sequences. J Comput Biol 7:203-214 doi:10.1089/10665270050081478</jrn> 


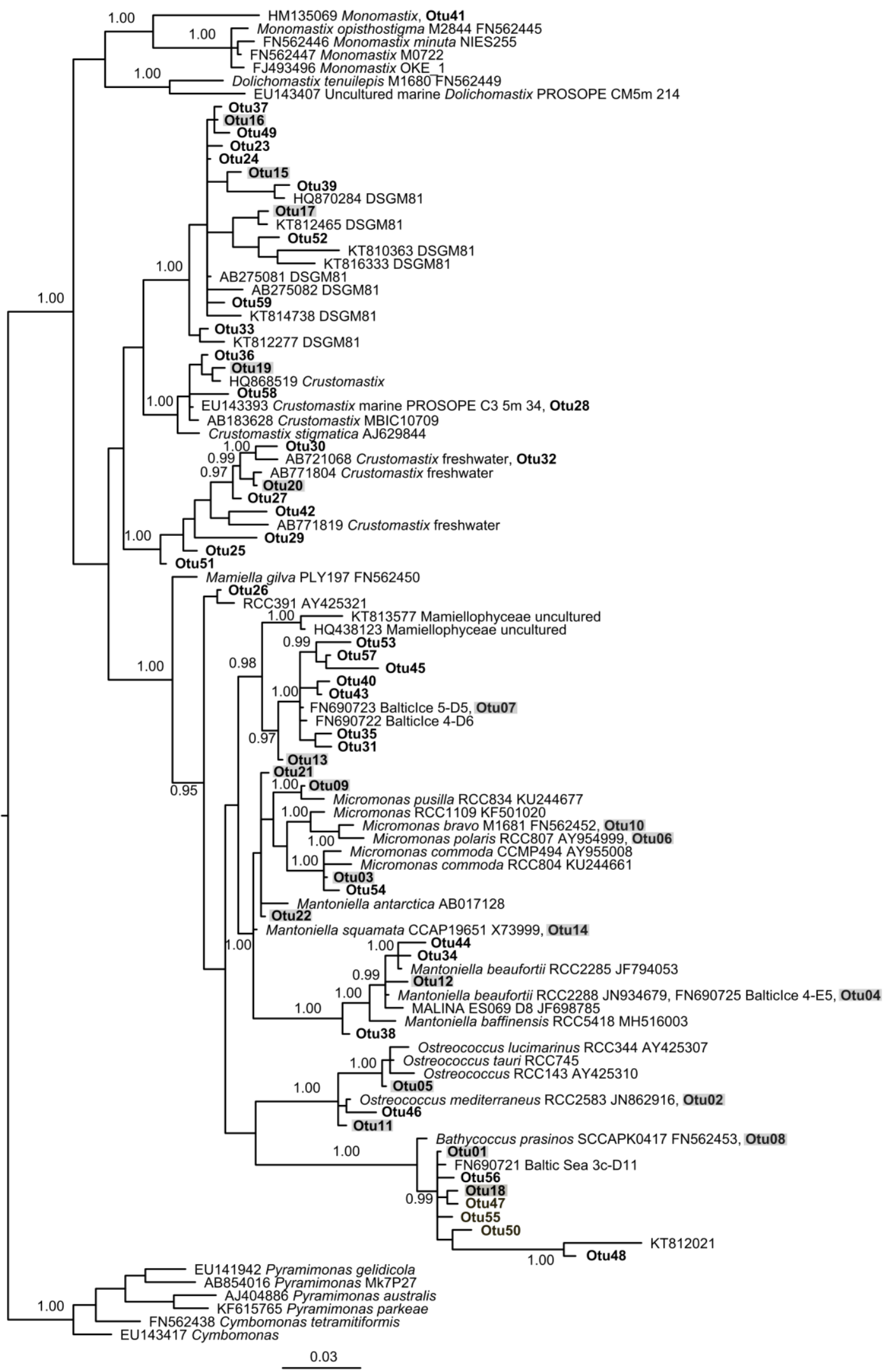

Fig. 1. Bayesian phylogenetic tree of Mamiellophyceae. Posterior probabilities $>0.95$ are shown on the branches. The operational taxonomic units (OTUs) of the current research are named Otu01, Otu02, etc., in decreasing order by their abundance in the total dataset, and are in bold. OTUs with $>100$ reads in total have a grey background 

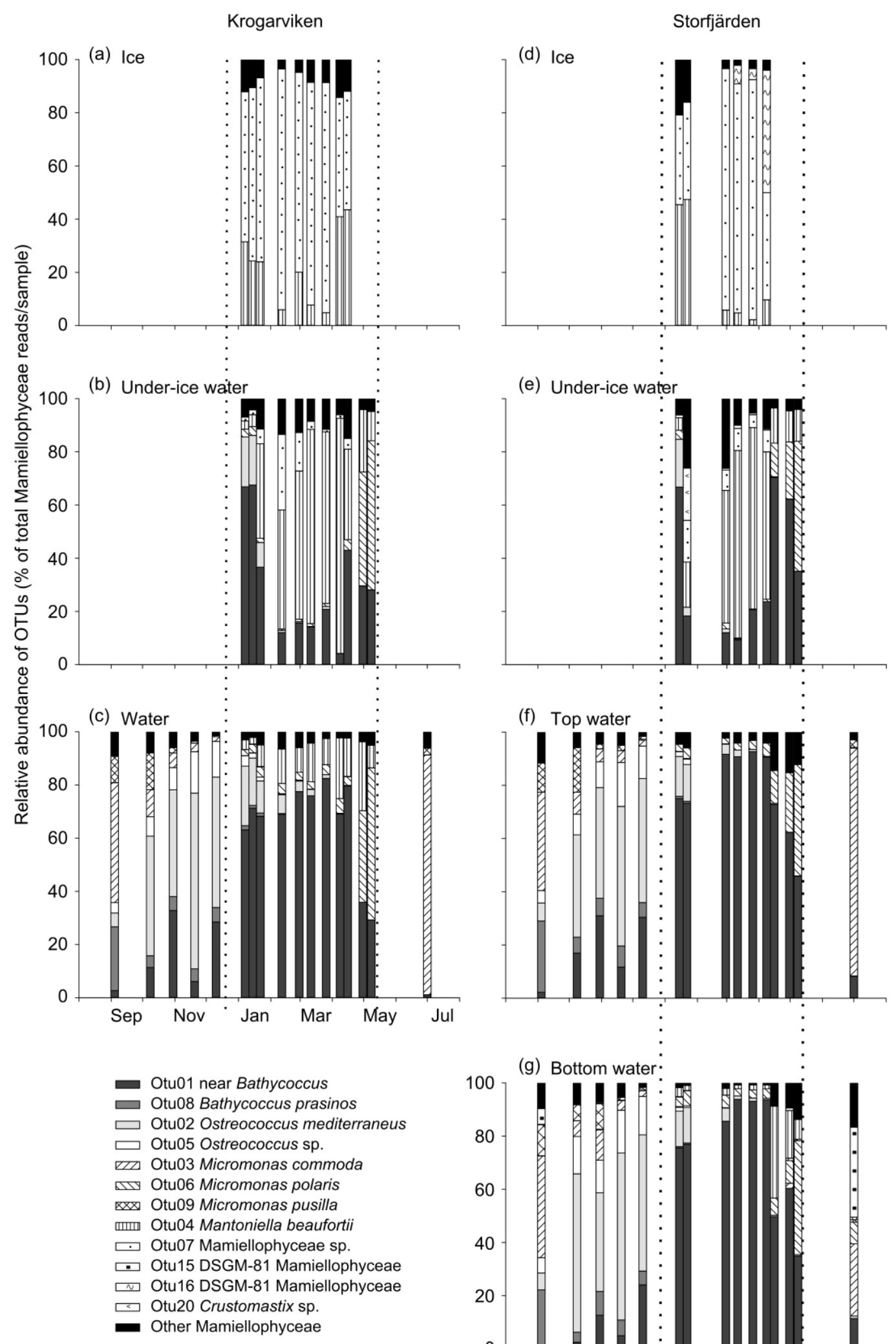

(e) Under-ice water
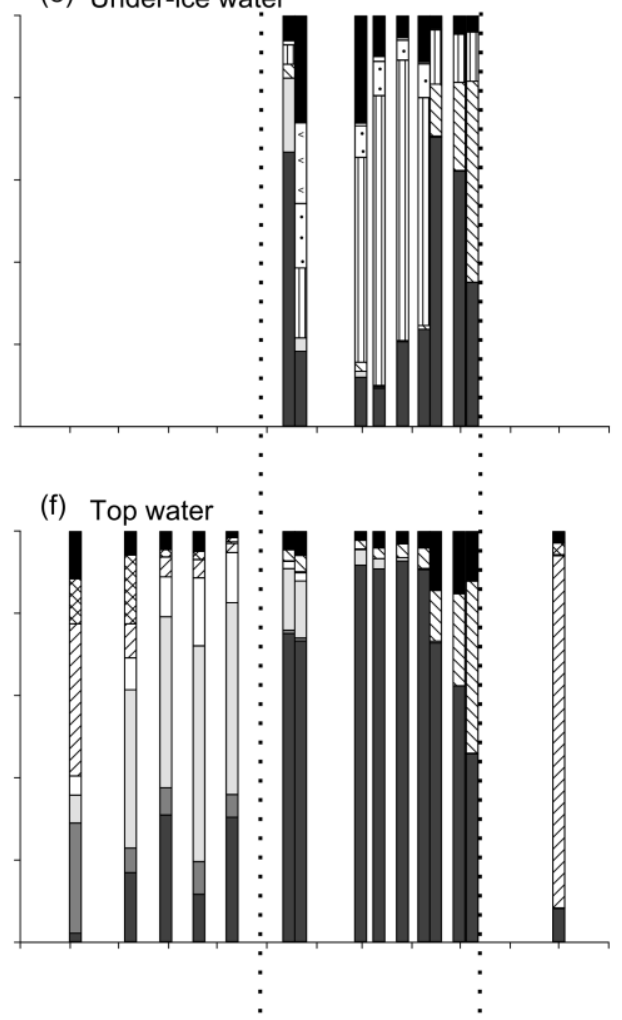

(g) Bottom water:

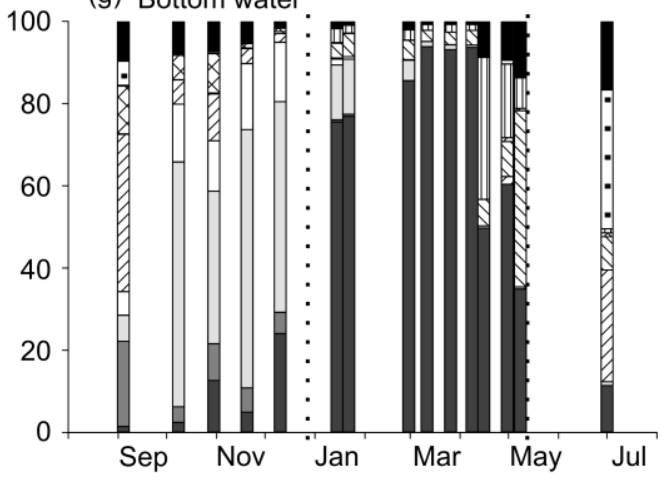

Fig. 2. Relative read abundance of the most abundant Mamiellophyceae operational taxonomic units in sea ice, under-ice water and in the water column at Krogarviken and Storfjärden throughout the sampling season. The water depth was $3 \mathrm{~m}$ at Krogarviken and ca. $30 \mathrm{~m}$ at Storfjärden. The top water was 0 to $15 \mathrm{~m}$ and the bottom water 15 to $30 \mathrm{~m}$ at Storfjärden. The time between dotted lines represents the ice-covered season 
(a) Storfjärden top water

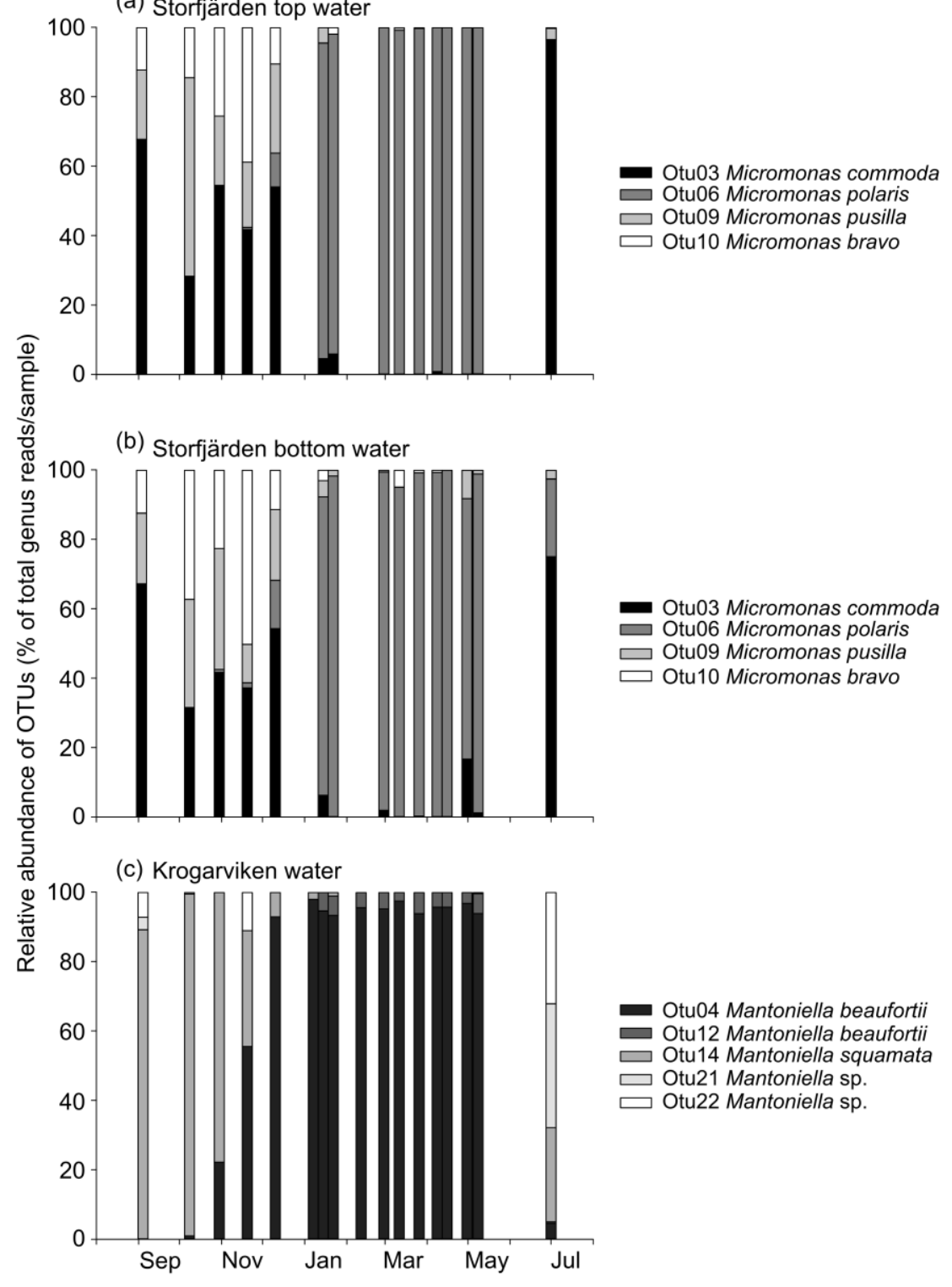

Fig. 3. Relative read abundance of Micromonas and Mantoniella operational taxonomic units in the water column 